\title{
Optical spectroscopy of the microquasar GRS 1758-258: a possible intermediate mass system?
}

\author{
Josep Martí ${ }^{1,3}$, Pedro L. Luque-Escamilla ${ }^{2,3}$, and Álvaro J. Muñoz-Arjonilla ${ }^{3}$ \\ 1 Departamento de Física, Escuela Politécnica Superior de Jaén, Universidad de Jaén, Campus Las Lagunillas s/n, A3, 23071 Jaén, \\ Spain \\ e-mail: jmarti@ujaen.es \\ 2 Departamento de Ingeniería Mecánica y Minera, Escuela Politécnica Superior de Jaén, Universidad de Jaén, \\ Campus Las Lagunillas s/n, A3, 23071 Jaén, Spain \\ e-mail: peter@ujaen.es \\ ${ }^{3}$ Grupo de Investigación FQM-322, Universidad de Jaén, Campus Las Lagunillas s/n, A3, 23071 Jaén, Spain \\ e-mail: ajmunoz@ujaen.es
}

Received 1 September 2016 / Accepted 28 October 2016

\begin{abstract}
Context. GRS 1758-258 is one of two prototypical microquasars towards the Galactic center direction discovered almost a quarter of a century ago. The system remains poorly studied in the optical domain due to its counterpart being a very faint and absorbed target in a crowded region of the sky.

Aims. Our aim is to investigate GRS $1758-258$ in order to shed light on the nature of the stellar binary components. In particular, the main physical parameters of the donor star, such as the mass or the spectral type, are not yet well constrained.

Methods. GRS 1758-258 has remained so far elusive to optical spectroscopy owing to its observational difficulties. Here, we use this traditional tool of stellar astronomy at low spectral resolution with a $10 \mathrm{~m}$ class telescope and a long slit spectrograph.

Results. An improved spectrum is obtained as compared to previous work. The quality of the data does not allow the detection of emission or absorption features but, nevertheless, we manage to partially achieve our aims comparing the de-reddened continuum with the spectral energy distribution expected from an irradiated disc model and different donor star templates.

Conclusions. We tentatively propose that GRS 1758-258 does not host a giant star companion. Instead, a main sequence star with mid-A spectral type appears to better agree with our data. The main impacts of this finding are the possibility that we are dealing with an intermediate mass system and, in this case, the prediction of an orbital period significantly shorter than previously proposed.
\end{abstract}

Key words. stars: individual: GRS 1758-258 - X-rays: binaries - infrared: stars

\section{Introduction}

GRS 1758-258, and its twin, 1E 1740.7-2942, are two microquasars with bipolar jets dominating the hard X-ray appearance of the sky in the vicinity of the Galactic center (Rodríguez et al. 1992; Mirabel et al. 1992). Their original discovery was one of the main results from the coded mask SIGMA telescope on board the Russian satellite GRANAT (Sunyaev et al. 1991; Goldwurm et al. 1994). For many years, controversy over their actual membership to the Milky Way could not be settled until clear evidence of morphological variability in their arc-minute extended jets was observed with modern radio interferometers in both systems (Martí et al. 2015; Luque-Escamilla et al. 2015). In the GRS 1758-258 case, the distance upper limit based on causality arguments is estimated to be $\sim 12 \mathrm{kpc}$. For discussion purposes, hereafter we assume a distance of $8.5 \mathrm{kpc}$ similar to that of the Galactic center.

Since GRS $1758-258$ is a very bright source in X-rays (see e.g. Soria et al. 2011), it has been easily studied in this energy range. However, this source has remained elusive to optical and near infrared analysis owing to the significant interstellar absorption towards it. The quest for a counterpart at these wavelengths has involved the hard work of many authors, starting with Bignami et al. (1991) and continued by, among others,
Martí et al. (1998), Rothstein et al. (2002), and Muñoz-Arjonilla et al. (2010). Only in recent times has a reliable counterpart candidate been finally identified based on both astrometric and photometric variability criteria (Luque-Escamilla et al. 2014). From X-ray spectral fitting, Soria et al. (2011) derive an equivalent hydrogen column density of $N_{\mathrm{H}} \simeq 1.6 \times 10^{22} \mathrm{~cm}^{-2}$. The corresponding extinction at visual wavelengths can be approximately estimated as $A_{V} \simeq 5.3 \times 10^{-22} \mathrm{~cm}^{-2} N_{\mathrm{H}}$ (Bohlin et al. 1978). This yields $A_{V}=8.9 \mathrm{mag}$, and this is the value that we adopt here for de-reddening of the spectral energy distribution (SED).

Confirmation of the stellar nature of GRS 1758-258 using spectroscopic tools with ground-based telescopes has remained a difficult task. This is because we are dealing with a very weak counterpart $(R=22.6 \pm 0.3, I=21.1 \pm 0.3$, Martí et al. 1998) in a crowded field, thus requiring the use of sub-arcsecond angular resolution as in Luque-Escamilla et al. (2014). Previous attempts by our team to secure an optical spectrum of GRS 1758-258 under average seeing conditions using a $10 \mathrm{~m}$-class telescope resulted in a poor quality result (Muñoz-Arjonilla et al. 2010). In this paper, we report the outcome of our second attempt to acquire optical spectroscopy of this microquasar under better observing conditions. Although the results are not yet optimal, new information is obtained that allows us to shed more light on our physical understanding of this system. 


\section{Observations}

GRS 1758-258 was observed with the Gran Telescopio Canarias (GTC) and its OSIRIS imager and spectrograph. The GTC run took place on the night of 1st June 2016 from the Observatorio del Roque de los Muchachos in the island of La Palma (Spain), under proposal code GTC23-15A. Observing conditions were clear and dark. The R300R grism was selected to acquire a low resolution $(\lambda / \Delta \lambda \simeq 350)$ spectrum. This choice was mandatory since we are dealing with a very faint and absorbed target whose brightness increases towards redder wavelengths. To avoid cosmic ray excesses, the total integration time was split into different observing blocks (OBs). Four of them could be executed with $1910 \mathrm{~s}$ of exposure each. The total on-source time thus amounted to approximately $2.1 \mathrm{~h}$ in service mode. The four frames acquisition took place during the UT time interval 01:42 to 03:54 centered around the target meridian transit. From La Palma, the source air mass does not drop below approximately 1.7 , even under these optimal conditions. Therefore, this GTC observation was a difficult one and, consequently, we requested very good seeing conditions for the execution of our OBs. At the time of observation, the seeing at the zenith was measured to be approximately 0.6 arcsec in the visual domain while, at the GRS 1758-258 air mass, this translated to a poorer 0.9 arcsecond value. Assuming a $\propto \lambda^{-1 / 5}$ scaling (Boyd 1978), this corresponds to a seeing value approaching 0.8 arcsec at our central wavelength $(0.8 \mu \mathrm{m}$ aprox.). This was judged as the minimum required to avoid most of the contamination from a nearby star located 1.5 arcsec away from our target by using a conservative slit width of 0.6 arcsec. The slit was also oriented along a position angle of $-16^{\circ}$, that is, orthogonal to the direction of the contaminating star brighter than our target by $\sim 1.6$ mag in the $R$-band. This object is also the same K giant star previously considered a GRS 1758-258 counterpart candidate, but later ruled out by Luque-Escamilla et al. (2014).

The raw frames were processed using the IRAF software package, including bias and spectroscopic flat field corrections. Wavelength calibration was secured by acquiring several arcs using different lamps ( $\mathrm{HgAr}, \mathrm{Ne}$ and $\mathrm{Xe}$ ) interleaved between the individual science frames. Target spectra were carefully extracted with sky background removed and later median combined to better exclude cosmic ray impacts. An approximate flux calibration was applied using one of the preferred GTC spectrophotometric standards (Ross640). Its uncertainty is conservatively estimated to be at the $\pm 0.1 \mathrm{mag}$ level. The slit transmission factor for the target narrow slit can be computed as:

$T_{\mathrm{x}}=\operatorname{erf}\left[\frac{0.5 \text { slit }}{\sqrt{2} \frac{\text { seeing }}{2.35482}}\right]=0.61$,

used to account for slit-losses in the target spectrum.

The final result is presented in Fig. 1 where a a highly absorbed featureless continuum is the most outstanding characteristic. To verify the flux calibration, at the $0.8 \mu \mathrm{m}$ wavelength the measured flux density is $4.5 \times 10^{-18} \mathrm{erg} \mathrm{s}^{-1} \mathrm{~cm}^{-2} \AA^{-1}$ with a signal-to-noise ratio of approximately 20 , corresponding to an $I$-band magnitude of $21.0 \pm 0.1$. This corresponds closely to previous broad-band photometry (Martí et al. 1998).

\section{Discussion}

Inspection of Fig. 1 shows that no intrinsic emission nor absorption feature is reliably detected on the GTC continuum, even in the $\mathrm{H} \alpha$ region where these features were more highly expected.

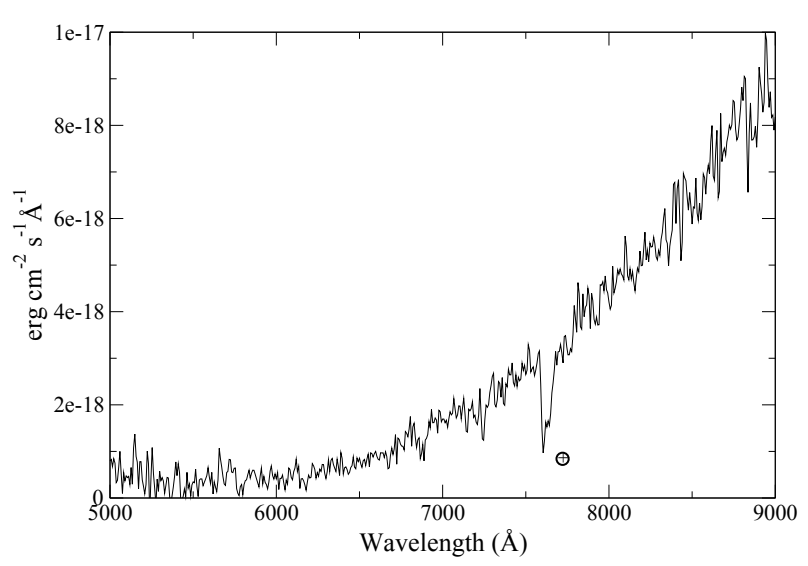

Fig. 1. GTC+OSIRIS spectrum of GRS 1758-258 obtained with the $\mathrm{R} 300 \mathrm{R}$ grism. The microquasar is barely detected at wavelengths shorter that $6000 \AA$. The prominent absorption feature at approximately $7600 \AA$ marked with the Earth symbol $\oplus$ is of telluric origin.

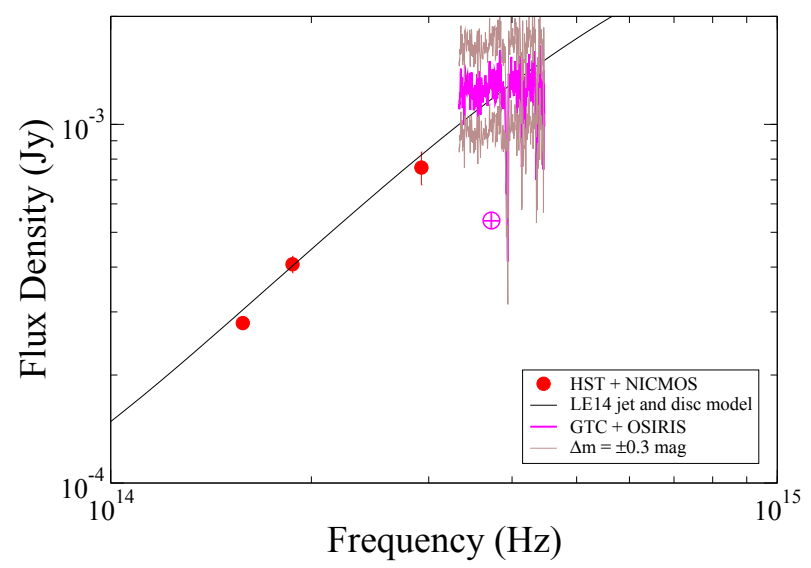

Fig. 2. De-reddened GTC spectrum of GRS 1758-258 plotted together with HST-NICMOS observations and the irradiated disc model by Luque-Escamilla et al. (2014) labelled as LE14. For comparison purposes, the GTC spectrum has also been plotted in brown scaled by a factor corresponding to variability and absolute flux uncertainty effects with $\Delta m= \pm 0.3 \mathrm{mag}$. The symbol $\oplus$ denotes the strongest telluric absorption feature.

The quality of the spectrum is thus not adequate to reveal stellar features that could enable a direct determination of the companion's spectral type. Nevertheless, the overall properties of the continuum level do provide some useful information when considered in the context of the system broad-band SED. For this purpose, the observed spectrum in Fig. 1 has been de-reddened using the $A_{V}$ value quoted in the introduction. When doing so, we have kept only the data points where the microquasar counterpart was detected with a signal-to-noise ratio higher than 5 $(\lambda \gtrsim 7000 \AA)$.

For comparison purposes, Fig. 2 shows the de-reddened GTC spectrum together with the irradiated disc model and the Hubble Space Telescope (HST) observations as reported in Luque-Escamilla et al. (2014). Their HST-NICMOS flux densities indicated a near-infrared spectrum rising with frequency according to $S_{v}=(0.13 \pm 0.01 \mathrm{mJy})\left[v / 10^{14} \mathrm{~Hz}\right]^{+1.7 \pm 0.2}$. This was interpreted as mostly due to emission from an irradiated accretion disc. In this context, the non-degenerated stellar companion was assumed to provide an almost negligible flux contribution, as is usual for low-mass X-ray binaries. However, extrapolation of this power law at higher optical frequencies does not 
appear now to be consistent with the much flatter GTC-OSIRIS spectrum (see again Fig. 2). The non-detection of a steeply rising spectrum in the GTC window forces us to revise our previous interpretation and points to the stellar continuum actually playing a role in the near infrared and optical domain.

The apparent mismatch between the HST-NICMOS points and the GTC spectrum in Fig. 2 could be due to the combined effect of the $\sim 0.2$ mag observed photometric variability (Luque-Escamilla et al. 2014) and the uncertainty in the flux calibration $( \pm 0.1 \mathrm{mag})$. Therefore, in this figure we also display the GTC data with scaling factors equivalent to $\Delta m= \pm 0.3$ mag. The lower brightness level would provide a much better continuity between the non simultaneous infrared and optical data. The uncertainty associated with the corresponding scaling factor $\left(10^{-0.4 \Delta m}\right)$ is approximately $5 \%$ for a GTC spectrum with signalto-noise ratio of $\sim 20$. Hereafter, we assume that this down-scaled spectrum is the most plausible data set for a physical discussion.

We carried out a tentative exploration of possible stellar companions using the Castelli and Kurucz atlas ${ }^{1}$. The same jet and irradiated disc model parameters used by Luque-Escamilla et al. (2014) were initially adopted, and part of the calculations were carried out using the Xspec software package from NASA. In order to achieve an acceptable fit, we tried different main sequence spectral types from $\mathrm{B}$ to $\mathrm{M}$ and different outer disc radii from $10^{3.5}$ to $10^{3}$ times the inner radius. Reducing the outer radius, always within the Xspec-allowed limits, removes part of the disc cooler regions that contribute significantly to optical and infrared emission. This enables a more dominating role of the stellar continuum in the $0.5-2 \mu \mathrm{m}$ region. The remaining parameters, including the $8.5 \mathrm{kpc}$ distance, were kept identical to those of Luque-Escamilla et al. (2014) since extensive modelling is beyond the approach of this observational paper.

To assess the quality of the synthetic SEDs, we computed their respective reduced $\chi_{\text {red }}^{2}$ value excluding the regions of strong telluric absorption. The result is presented in Fig. 3, where a minimum value of $\chi_{\text {red }}^{2}=0.94$ was achieved in the case of an $\mathrm{A} 5 \mathrm{~V}$ companion star and an outer radius $10^{3.05}$ times the inner value. For a total of 246 degrees of freedom (248 GTC used data points minus two explored parameters), the associated non-chance probability is greater than $70 \%$. The corresponding SED fit is represented by the solid line in Fig. 4. The error bars shown take into account both the rms of the GTC spectrum and the 5\% uncertainty in the adopted scaling factor. Neither varying the stellar template by a few spectral sub-types nor changing the scaling factor by a few percentage have a strong effect on the best fit result. Spectral types later than A5V provide progressively higher $\chi_{\text {red }}^{2}$ values and lower non-chance probabilities (see Fig. 3 and Table 1). Although we cannot fully rule these cooler stars out, the suggestion of an A-type main sequence companion is the best supported by current GTC data.

Our tentative interpretation points to the stellar companion and the accretion disc comparably contributing to the flux in the optical domain, where the emission from the synchrotron jet would be negligible. This can be better appreciated in Fig. 5 that displays a zoomed out version of Fig. 4 extending from radio to $\mathrm{X}$-ray energies.

It is also important to stress that a mid-A spectral type would render GRS $1758-258$ a system with possible connections with the sub-class of intermediate mass X-ray binaries (IMXBs, see e.g. Tauris \& van den Heuvel 2006, for a review). For instance, GRS 1758-258 would fit in the spectral type range covered by

\footnotetext{
1 http://www.stsci.edu/hst/observatory/crds/castelli_
} kurucz_atlas.html

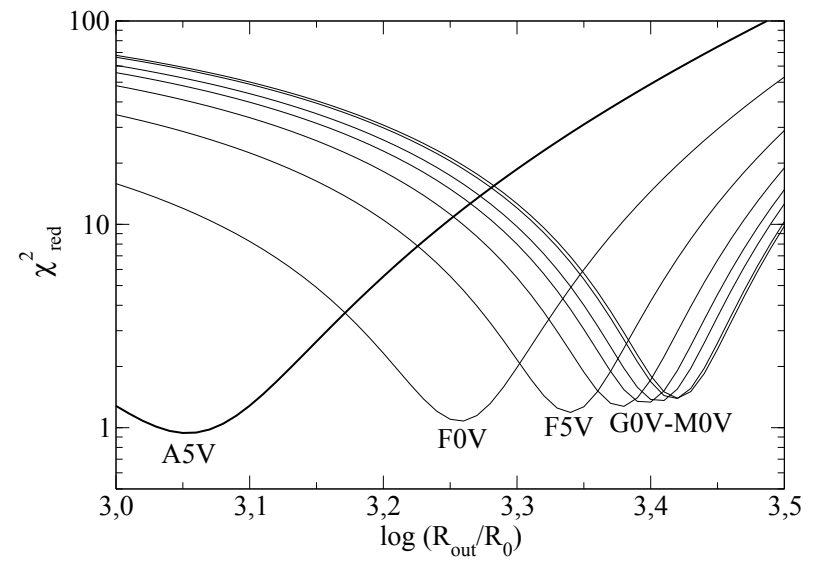

Fig. 3. Values of $\chi_{\text {red }}^{2}$ for the range of outer radii and spectral types explored in this paper. The SED assuming an A5V companion star provided the smallest value of this statistical indicator. The curves corresponding to B and early-type A stars are above and outside the range of this plot.

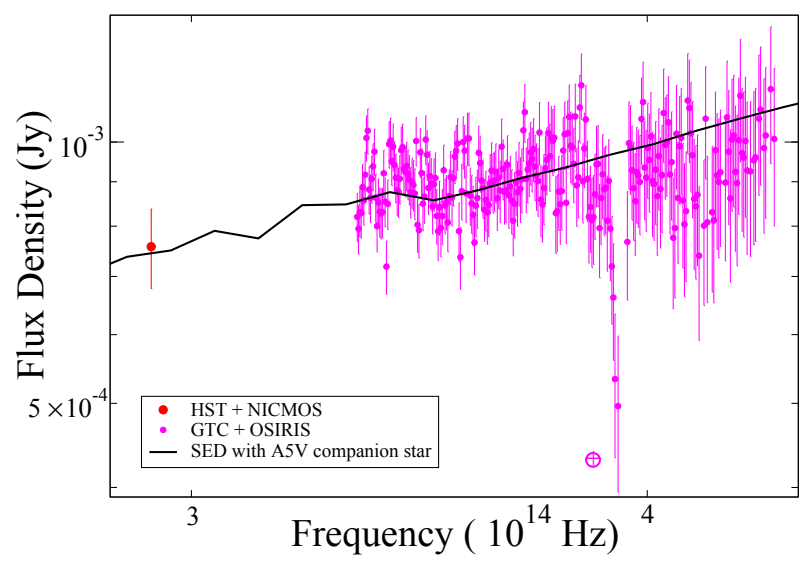

Fig. 4. Detailed view of the de-reddened GTC spectrum as a function of frequency and including estimated error bars. The solid line is the SED fit corresponding to the $\chi_{\text {red }}^{2}$ minimum in Fig. 3 obtained for an A5V star. The symbol $\oplus$ marks the strongest telluric absorption that is excluded from the fit.

Table 1. Goodness of the SED fit in the GTC domain.

\begin{tabular}{cccc}
\hline $\begin{array}{c}\text { Spectral type } \\
\text { of companion }\end{array}$ & $\log \left[R_{\text {out }} / R_{0}\right]$ & $\chi_{\text {red }}^{2}$ & $\begin{array}{c}\text { Non-chance } \\
\text { probability }^{a}\end{array}$ \\
\hline A5V & 3.05 & 0.94 & $73.37 \%$ \\
F0V & 3.26 & 1.08 & $18.91 \%$ \\
F5V & 3.34 & 1.19 & $2.25 \%$ \\
G0V & 3.38 & 1.27 & $0.23 \%$ \\
G5V & 3.40 & 1.34 & $0.03 \%$ \\
K0V & 3.41 & 1.36 & $0.01 \%$ \\
K5V & 3.42 & 1.39 & $0.00 \%$ \\
M0V & 3.42 & 1.40 & $0.00 \%$ \\
\hline
\end{tabular}

Notes. ${ }^{(a)}$ For 246 degrees of freedom.

some black hole IMXBs such as GRO J1655-40 (spectral type F5IV, Beer \& Podsiadlowski 2002) and 4U 1543-47 (spectral type A2V, Orosz et al. 1998).

The suggestion that the stellar companion of GRS 1758-258 is a main sequence star instead of a late-type giant implies a re-interpretation of reported X-ray periods of approximately18 d previously proposed to represent the orbital cycle 


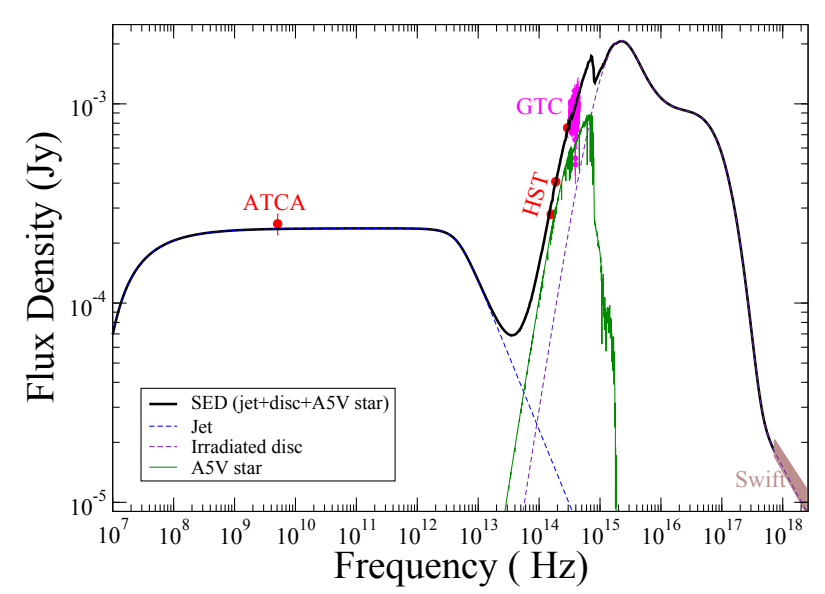

Fig. 5. Same kind of plot as in Fig. 4 but with the SEDs extending from radio to the X-ray domain. The solid line is the same SED best fit using an $\mathrm{A} 5 \mathrm{~V}$ star in addition to the flat spectrum radio jet and irradiated disc components. These components are also plotted separately and identified in the legend.

(Smith et al. 2002; Levine et al. 2011), but not yet confirmed. Indeed, mass feeding of the compact object by Roche lobe overflow would not be feasible for such a long orbital period with a non-giant star. The required orbital period needs to be significantly shorter, as illustrated in Fig. 6. Here, the Eggleton (1983) approximation for the Roche lobe radius has been used:

$r_{\mathrm{L}}=a \frac{0.49 q^{2 / 3}}{0.6 q^{2 / 3}+\log \left(1+q^{1 / 3}\right)}$,

where $a$ is the system separation (circular orbit assumed) and $q=M_{\mathrm{X}} / M_{*}$ is the mass ratio of the primary over the secondary star (the compact object in our case). Adopting stellar masses and radii of normal stars from Chap. 15 in Cox (2000), together with Kepler's third law, the expected orbital period can be easily computed. As a result, an orbital period in the range $0.5-1.0 \mathrm{~d}$ is anticipated even accounting for an uncertainty of some spectral sub-types in our SED considerations (see Fig. 6). In this context, the $18 \mathrm{~d}$ period could be perhaps due to a precession motion of the disc. Precession periods in X-ray binaries are usually found to be within $10-10^{2}$ times the orbital value (Larwood 1998). The ratio 1 to 18 would fit nicely within this range. Moreover, a shorter orbital period would also favour a possible disc truncation in agreement with the smaller outer radius provided by our tentative SED fit.

\section{Conclusions}

The optical counterpart of the microquasar GRS 1758-258 has been studied with optical spectroscopy using the GTC. Unfortunately, no emission nor absorption lines were detected. Despite the challenging difficulties of the observation, the resulting data allow us to put some constraints on the nature of the donor star in the system. The shape of the continuum does not agree with a dominant contribution of the accretion disc in the optical domain as proposed in previous work. Instead, the donor star is found to be more consistent with a main sequence object of mid-A spectral type and accounting for a significant fraction of the optical flux. If this is correct, GRS 1758-258 could be connected with the sub-class of IMXBs. Finally, a short orbital period in the $0.5-1.0 \mathrm{~d}$ range is predicted in order to allow Roche lobe overflow. In this context, previously reported $\mathrm{X}$-ray periods of approximately $18 \mathrm{~d}$ would correspond to a

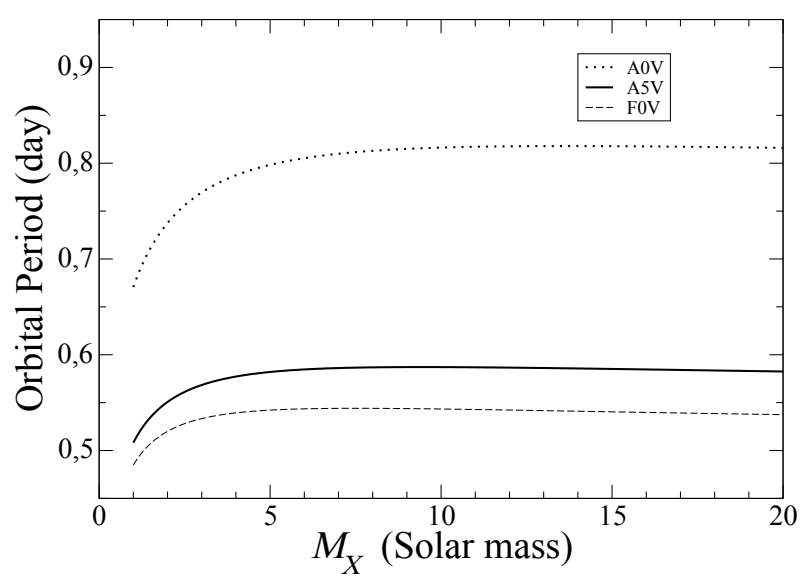

Fig. 6. Estimated orbital period of GRS 1758-258 as a function of plausible masses $M_{\mathrm{X}}$ of the compact companion and a Roche lobe filling star \pm 5 spectral sub-types around $\mathrm{A} 5 \mathrm{~V}$. The assumed mass and radius of an A5V star are $R_{*}=2 M_{\odot}$ and $R_{*}=1.7 R_{\odot}(\operatorname{Cox} 2000)$.

physical mechanism other than the orbital cycle such as precession. Improved observations (e.g. with adaptive optics or an even larger telescope) will be required to finally reveal photospheric features in the GRS 1758-258 spectrum.

Acknowledgements. Based on observations made with the Gran Telescopio Canarias (GTC), installed in the Spanish Observatorio del Roque de los Muchachos of the Instituto de Astrofísica de Canarias, in the island of La Palma. This work was supported by grant AYA2013-47447-C3-3-P from the Spanish Ministerio de Economía y Competitividad (MINECO), and by the Consejería de Economía, Innovación, Ciencia y Empleo of Junta de Andalucía under excellence grant FQM-1343 and research group FQM-322, as well as FEDER funds. IRAF is distributed by the National Optical Astronomy Observatory, which is operated by the Association of Universities for Research in Astronomy (AURA) under a cooperative agreement with the National Science Foundation.

\section{References}

Beer, M. E., \& Podsiadlowski, P. 2002, MNRAS, 331, 351

Bignami, G. F., Caraveo, P. A., Mereghetti, S., et al. 1991, The Messenger, 66, 10

Bohlin, R. C., Savage, B. D., \& Drake, J. F. 1978, ApJ, 224, 132

Boyd, R. W. 1978, J. Opt. Soc., 68, 877

Cox, A. N. 2000, Allen's astrophysical quantities (New York: AIP Press, Springer)

Eggleton, P. P. 1983, ApJ, 268, 368

Goldwurm, A., Cordier, B., Paul, J., et al. 1994, Nature, 371, 589

Larwood, J. 1998, MNRAS, 299, L32

Levine, A. M., Bradt, H. V., Chakrabarty, D., Corbet, R. H. D., \& Harris, R. J. 2011, ApJS, 196, 6

Luque-Escamilla, P. L., Martí, J., \& Muñoz-Arjonilla, Á. J. 2014, ApJ, 797, L1

Luque-Escamilla, P. L., Martí, J., \& Martínez-Aroza, J. 2015, A\&A, 584, A122

Martí, J., Mereghetti, S., Chaty, S., et al. 1998, A\&A, 338, L95

Martí, J., Luque-Escamilla, P. L., Romero, G. E., Sánchez-Sutil, J. R., \& Muñoz-Arjonilla, Á. J. 2015, A\&A, 578, L11

Mirabel, I. F., Rodríiguez, L. F., Cordier, B., Paul, J., \& Lebrun, F. 1992, Nature, 358,215

Muñoz-Arjonilla, A. J., Martí, J., Luque-Escamilla, P. L., et al. 2010, A\&A, 519, A15

Orosz, J. A., Jain, R. K., Bailyn, C. D., McClintock, J. E., \& Remillard, R. A. 1998, ApJ, 499, 375

Rodríguez, L. F., Mirabel, I. F., \& Martí, J. 1992, ApJ, 401, L15

Rothstein, D. M., Eikenberry, S. S., Chatterjee, S., et al. 2002, ApJ, 580, L61

Smith, D. M., Heindl, W. A., \& Swank, J. H. 2002, ApJ, 578, L129

Soria, R., Broderick, J. W., Hao, J., et al. 2011, MNRAS, 415, 410

Sunyaev, R., Churazov, E., Gilfanov, M., et al. 1991, A\&A, 247, L29

Tauris, T. M., \& van den Heuvel, E. P. J. 2006, in Compact stellar X-ray sources, eds. W. H. G. Lewin, \& M. van der Klis, Camb. Astrophys. Ser., 39, 623 